\title{
Growth and Characterization of Pencil-Like ZnO Nanowires in the Presence of a Disturbance in Boundary Layer
}

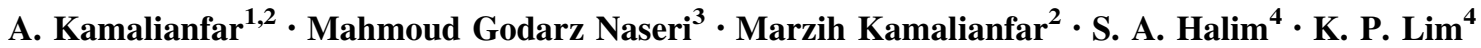

Received: 25 December 2015/Revised: 10 February 2016/Published online: 5 May 2016

(C) The Chinese Society for Metals and Springer-Verlag Berlin Heidelberg 2016

\begin{abstract}
Pencil-like zinc oxide $(\mathrm{ZnO})$ nanowire was synthesized on $\mathrm{Si}(111)$ substrate through a simple vapor phase method using a mixture of zinc oxide and graphite as the source material. The source inside a quartz tube created a $\mathrm{Zn}$-rich vapor that facilitated the formation and growth of $\mathrm{ZnO}$ nanowires. Field emission scanning electron microscopic studies indicated that pencil-like $\mathrm{ZnO}$ nanowires had a size of the range from 50 to $150 \mathrm{~nm}$ in diameter and several microns in length. X-ray diffraction was used to investigate the crystal structure of $\mathrm{ZnO}$ nanowires. Raman scattering and photoluminescence were applied to characterize the optical properties of the pencils. The growth mechanism of the nanopencils was discussed based on the growth conditions.
\end{abstract}

KEY WORDS: Zinc oxide; Nanowires; Vapor-phase transport; Pencil-like

\section{Introduction}

Zinc oxide $(\mathrm{ZnO})$ with a direct band gap of $3.37 \mathrm{eV}$ and large exciton binding energy of $60 \mathrm{meV}$ at room temperature (RT) is a promising material for many applications in electronic and optical wave guide devices such as solar cells, gas sensor ultraviolet (UV) light-emitting devices and so on.

Available online at http://link.springer.com/journal/40195

A. Kamalianfar

kamalianfar.ahmad@gmail.com

1 Department of Physics, Faculty of Science, Farhangian University, Shahid Rajaee Pardis, Shiraz 71456-15515, Iran

2 Ministry of Education, Fars Education, Shiraz 74347-86187, Iran

3 Department of Physics, Faculty of Science, Malayer University, Malayer 65719-95863, Iran

4 Department of Physics, Faculty of Science, University Putra Malaysia, UPM, 43400 Serdang, Selangor, Malaysia
In recent years, fabrication and characterization of $\mathrm{ZnO}$ micro- and nanostructures have attracted remarkable attention due to their outstanding structure- and size-dependent optical and electrical properties [1-4]. Because the properties of materials depend much on their sizes and shapes, it is necessary to study various sizes and morphographies of $\mathrm{ZnO}$ nanostructures.

The controlled synthesis of the nanostructures, which is incorporated into functional nanodevices, is a rather challenging issue for desired size, shape, and orientation. One way to solve this problem is to synthesize various nanostructures by various methods. The growth of $\mathrm{ZnO}$ nanostructures has been reported by various methods, including hydrothermal process [5], laser ablation [6] vapor phase methods [7, 8], and sol-gel [9]. Most of modifications in the basic system geometry aim to allow for better control of the reaction conditions in the vapor phase. Accordingly, in the present work, we demonstrate a simple approach to synthesizing $\mathrm{ZnO}$ nanowires using a vapor-phase transport method under air atmosphere to reduce energy consumption. In addition, by changing the system geometry, we studied the effects of a wall (a piece of aluminum foil) inside a quartz tube and through $\mathrm{Zn}$ vapor direction on the 
morphological and optical properties of the as-grown sample.

\section{Experimental}

Silicon (111) was ultrasonically cleaned with ethanol and deionized water for $30 \mathrm{~min}$. A mixture of zinc oxide and commercial graphite powder in a molar ratio of $1: 1$ was loaded onto the alumina boats which were transferred to a narrow-bore ( $2 \mathrm{~cm}$ in diameter, $50 \mathrm{~cm}$ in length) quartz tube in the central of an electric furnace tube. The substrate and source were placed at different temperature zones in the 15-cm-diameter quartz tube.

Two similar quartz tubes were placed next to each other in the electric furnace tube. One is a normal quartz tube, and the other is a modified quartz tube. A wall of height $1 \mathrm{~cm}$ (an aluminum foil) was placed directly between the source and substrate as shown in Fig. 1. The furnace was heated up to $910{ }^{\circ} \mathrm{C}$ and was maintained at this temperature for $15 \mathrm{~min}$.

The electric furnace was naturally cooled down to the room temperature. The crystal structure and morphology of the samples were investigated using a field emission scanning electron microscopy (FESEM, JSM 6700) and $\mathrm{X}$-ray diffraction (XRD, Shimadzu 6000) employing $\mathrm{Cu} K \alpha$ $(0.154 \mathrm{~nm})$ radiation. The optical properties of the samples were characterized using a laboratory setup with a pulsed xenon lamp (LS-55, Xe lamp) and Raman (Jobin-Yvon Horiba HR 800 UV) spectroscopy.

\section{Results and Discussion}

One sample (sample S1) was grown by the modified tube and a reference sample by the normal tube (sample S2). The morphology of the as-grown samples was examined using an FESEM. Figure 2 a shows a low magnification FESEM image of sample S1. It can be seen that the pencillike nanowires are grown in a very high density. The high magnification SEM images (Fig. 2b, c) indicate that each

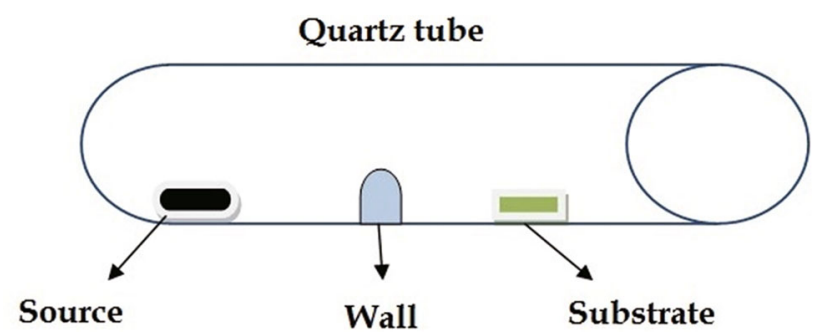

Fig. 1 Schematic of the setup used for the growth of the $\mathrm{ZnO}$ nanowires pencil consists of a pen-nib and holder. The diameters of the pen holders and pen-nibs are about $150 \pm 50$ and $50 \pm 10 \mathrm{~nm}$, respectively. The pencils have the lengths of about several microns.

Figure $2 \mathrm{~d}$ shows another typical morphology of $\mathrm{ZnO}$ nanostructure grown in the normal tube with no aluminum wall (sample S2). Versatile morphologies including nail, spear and rod nanostructures can be seen clearly. They have a range from 100 to $250 \mathrm{~nm}$ in diameter and several microns in length. The results demonstrate that the shapes of the products depend on the growth conditions. Placement of the wall between the source and the substrate may result in significantly lower vapor pressure of the reactants and thus better ordering and uniform shape of the nanostructures. To investigate the crystal structure of the samples, the XRD pattern of the pencil-like $\mathrm{ZnO}$ was examined. It was confirmed that the crystalline phase of the sample is the wurtzite structure (hexagonal with space group $P 63 m c$ ).

All diffraction peaks were indexed as shown in Fig. 3 and were in agreement with JCPDS 36-1451. The cell constants of $a(0.325 \mathrm{~nm})$ and $c(0.521 \mathrm{~nm})$ are quite similar to those of pure $\mathrm{ZnO}$. No peaks from $\mathrm{Zn}$ or other impurities were detected.

The (002) peak was chosen to estimate the crystallite size $D$ from the maximum intensity of the full width at half maximum (FWHM, $\beta$ ) related to the films with different thicknesses. To calculate the crystallite size $D$, we used Debye-Scherrer's formula:

$D=\frac{0.94 \lambda}{\beta \cos (\theta)}$,

where $\lambda$ is $\mathrm{X}$-ray wavelength $(0.15405 \mathrm{~nm}$ in this case $), \beta$ is the diffraction line broadening at FWHM of the peak, and $\theta$ is the peak position. The results are presented in Table 1.

Studies of the luminescence of $\mathrm{ZnO}$ crystals indicate that two emission bands can be observed in the photoluminescence spectra: One is in the ultraviolet (UV) region around $380 \mathrm{~nm}$ or $3.25 \mathrm{eV}$, and the other a broad green band between 500 and $530 \mathrm{~nm}$ or $2.35-2.50 \mathrm{eV}$. It is generally accepted that the UV emission of $\mathrm{ZnO}$ can be attributed to the mixture of free exciton and other impurityrelated transitions at the near-band edge (NBE) [10]. The band structure calculations propose that the deep-level green band emission (DLE) is attributed to singly ionized oxygen vacancy in $\mathrm{ZnO}$. It came from recombination of a photogenerated hole with the singly ionized charge state of this defect [11]. The room temperature photoluminescence spectra of the products are shown in Fig. 4. The strong UV peaks (also called the near-band-edge (NBE) emission) at $381 \mathrm{~nm}$ with a narrow FWHM of $21 \mathrm{~nm}$ for sample S1 and a weaker one at $384 \mathrm{~nm}$ for sample S2 are observed in the 

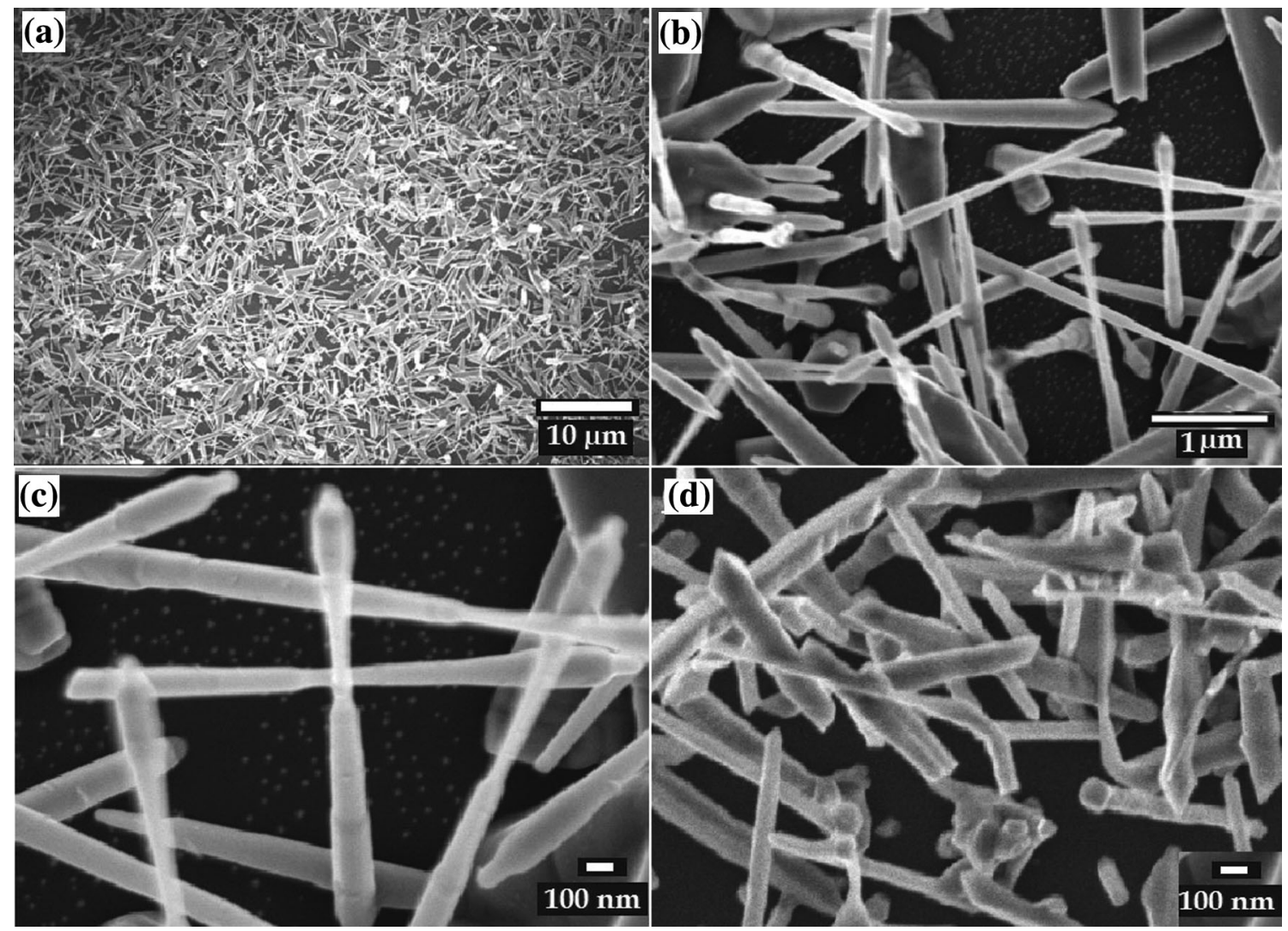

Fig. 2 Typical FESEM images of the $\mathrm{ZnO}$ nanopencil grown in the modified tube a-c and $\mathrm{ZnO}$ nanostructure grown in the normal tube $\mathbf{d}$

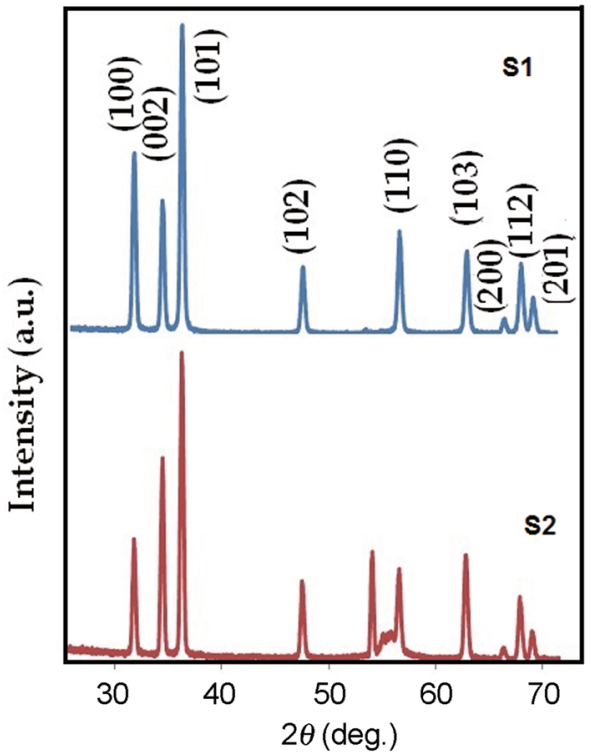

Fig. 3 XRD patterns of the $\mathrm{ZnO}$ nanowires grown by modified (sample S1) and normal (sample S2) vapor method

spectra. The differences in the intensity and position of the UV peaks are due to the different growth conditions, the effect on the relative contributions of free exiton emission and phonon. In the case of the structure of nanopencils, the wall in the modified tube decreases the boundary layer thickness and causes the reduction in strain defects as well as improves the crystallize size.

For both samples S1 and S2, a dominant visible emission manifested as a broad feature in the range of $450-550 \mathrm{~nm}$. Although the exact origin of this broad visible band emission is still an open question, it is suggested that it corresponds to the singly ionized oxygen vacancy in $\mathrm{ZnO}$, the fast reaction formation process and the large surface-to-volume ratio $[12,13]$. The ratio of the UV and visible intensity (UV/DLE) provides some indication of the material quality and comparison of optical properties between samples [14]. The high UV/DLE ratio of sample S1 compared with that of sample S2, in addition to the narrow UV peak and the decrease in the peak intensity of the green emission, could be the result of high crystalline quality of the pencils. In addition, the DLE is enhanced while the UV emission is suppressed due to the increase in both the oxygen vacancies and zinc interstitials of sample S2.

Raman scattering spectroscopy is a technique used to observe low-frequency modes such as rotation and vibration in a system. When a laser light interacts with molecular vibrations or phonons, the frequency of photons is shifted up or down. The change in the photon frequency 
Table 1 Comparison of position, height, FWHM, and crystallite size of (002) diffraction peaks

\begin{tabular}{lllll}
\hline Sample & Position angle, $2 \theta$ & Height (CTs) & FWHM, $\beta$ & Crystallite size (nm) \\
\hline S1 & 34.49 & 1485 & 0.34 & 49 \\
S2 & 34.45 & 1725 & 0.39 & 43 \\
\hline
\end{tabular}

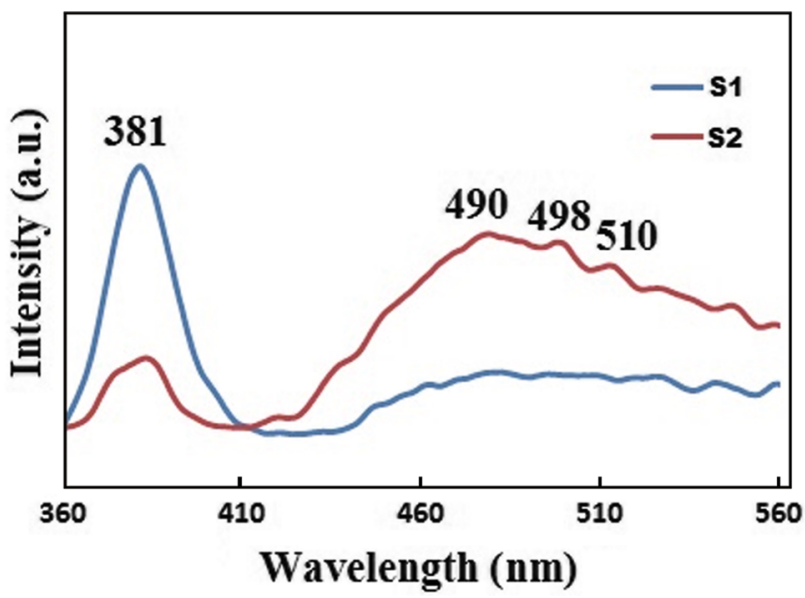

Fig. 4 Room-temperature photoluminescence spectra, the $\mathrm{ZnO}$ nanopencil grown in the modified tube (sample S1), and the $\mathrm{ZnO}$ nanostructure grown in the normal tube (sample S2)

provides information about molecular vibrations that can be used for structural defection and crystal perfection of the system.

Based on the vibrational modes, the thermal and electrical properties of a single crystal, such as the wurtzite $\mathrm{ZnO}$ structure, can be investigated.

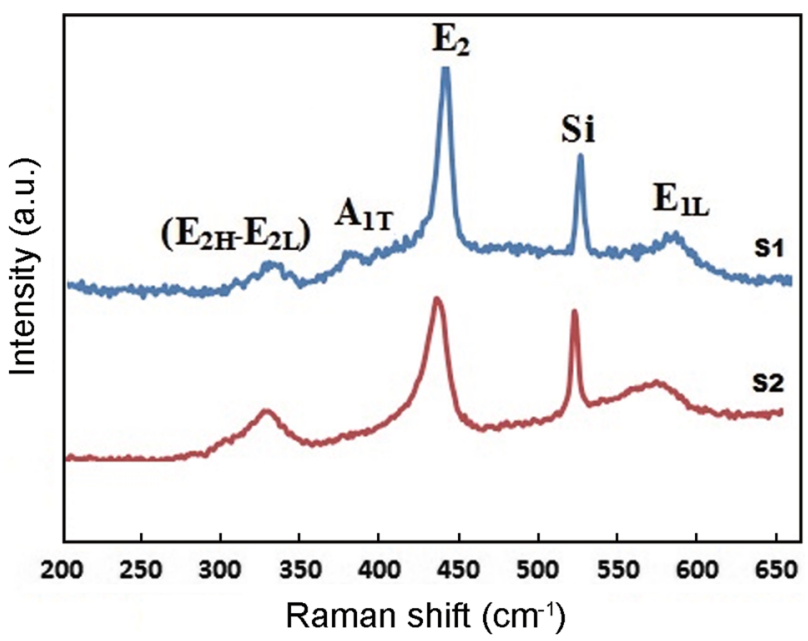

Fig. 5 Raman spectra excited by 514-nm line, the $\mathrm{ZnO}$ nanopencil grown in the modified tube (sample $\mathrm{S} 1$ ), and the $\mathrm{ZnO}$ nanostructure grown in the normal tube (sample $\mathrm{S} 2$ )
Figure 5 shows the characteristic Raman spectra features of both samples $\mathrm{S} 1$ and $\mathrm{S} 2$. Several bands related to Raman-active phonon modes of wurtzite $\mathrm{ZnO}$ nanostructure with C6 V symmetry can be observed. The strong Si peak is coming from the substrate.

The $E_{2}$ (high) phonon frequency at $437 \mathrm{~cm}^{-1}$ is the intrinsic characteristic of hexagonal wurtzite $\mathrm{ZnO}$ [15]. The FWHM of the $E_{2}$ (high) mode decreased from sample S2 to $\mathrm{S} 1$. Therefore, this result and the PL results indicate that the crystallinity of the nanopencils is higher than that of the nanowires in sample $\mathrm{S} 2$.

The $E_{2}$ (high) mode of the wurtzite $\mathrm{ZnO}$ crystal structure would shift to a lower frequency under internal strains resulting in different growth conditions. The $E_{2}$ (high) mode has been blueshifted $\left(434 \mathrm{~cm}^{-1}\right)$ for sample $\mathrm{S} 2$ compared with bulk $\mathrm{ZnO}\left(437 \mathrm{~cm}^{-1}\right)$. Using the below equation [16], the stress value of sample $\mathrm{S} 2$ is found to be $0.454(\mathrm{GPa})$.

$\Delta \omega=4.4 \sigma$.

where $\sigma$ is the stress of nanostructures in the $c$-axis direction in GPa and $\Delta \omega$ is the shift of $E_{2}$ (high) mode in $\mathrm{cm}^{-1}$.

The forbidden mode attributed to the $E_{2}$ (high) $-E_{1}$ (low), or multi-phonon process mode is located at $331 \mathrm{~cm}^{-1}$ frequency of second order. This mode can only be found when the $\mathrm{ZnO}$ is a single crystal [17].

The peaks at $580 \mathrm{~cm}^{-1}$ corresponding to the $E_{1}$ (LO) vibration mode are related to impurities and formation of defects such as oxygen vacancies, zinc interstitial and free carriers [18]. It seems that the $E_{1}$ (LO) mode is broad and is enhanced by disorder [19].

The reason of the low intensity of them is due to more ordered wurtzite structures as seen in the peak at $438 \mathrm{~cm}^{-1}$. Additionally, Fig. 5 shows a peak at $380 \mathrm{~cm}^{-1}$ corresponding the A1 (TO) mode. This peak usually originated from structure or doping induced by disorder in the $\mathrm{ZnO}$ substrate.

\section{Theory of the Growth Mechanism through a Boundary Layer}

As known, one-dimensional diffusion of a gas is written by Fick's first law: 
$J=-D \frac{\mathrm{d} c(x)}{\mathrm{d}(x)}$,

where $J$ is the diffusion flux (amount of substance per unit area per unit time), $D$ is the intrinsic diffusion coefficient, and $c$ is the concentration at $x$.

In the vapor phase transport (VPT) method, gaseous species move from the source region to the substrates through a layer of reduced velocity vapor, called boundary layer (Fig. 6). Under certain conditions adjacent to the wall, separation of the boundary layer can occur due to excessive momentum loss near the wall. In this condition, the gaseous species try to move downstream against increasing pressure which is called adverse pressure gradient. Somewhere around the top of the wall, the fluid particles in the boundary layer are separated from the wall and are driven in the upstream direction. The influence of the boundary layer separation on the surface reaction and adsorption, results in the overall growth rate and different morphologies [20].

To explain the growth mechanism of the obtained nanostructure (pencil-like), the idealized growth habit of the $\mathrm{ZnO}$ crystal can be used. Vapor-solid (VS) growth process is suggested for the formation of this kind of crystal [21, 22].

The reactions that govern VPT growth of $\mathrm{ZnO}$ nanostructures are as follows:

$$
\begin{aligned}
& \mathrm{ZnO}_{(\mathrm{s})}+\mathrm{C}_{(\mathrm{s})} \rightarrow \mathrm{Zn}_{(\mathrm{g})}+\mathrm{CO}_{(\mathrm{g})}, \\
& \mathrm{ZnO}_{(\mathrm{s})}+\mathrm{CO}_{(\mathrm{g})} \rightarrow \mathrm{CO}_{2(\mathrm{~g})}+\mathrm{Zn}_{(\mathrm{g})}, \\
& \mathrm{Zn}_{(\mathrm{g})}+\mathrm{O}_{2(\mathrm{~g})} \rightarrow \mathrm{ZnO}_{(\mathrm{g})}, \\
& \mathrm{ZnO}_{(\mathrm{g})} \rightarrow \mathrm{ZnO}_{(\mathrm{s})} .
\end{aligned}
$$

The melting point of solid $\mathrm{ZnO}$ is reduced (from 1975 to $910{ }^{\circ} \mathrm{C}$ ) by graphite. The gases produced by the first reaction are $\mathrm{Zn}(\mathrm{g})$ and $\mathrm{CO}(\mathrm{g})$. The $\mathrm{Zn}$ vapors transfer from high-temperature zones into the low-temperature zones and deposit on the substrate. The facet with higher surface energy is usually small in area, while the lower energy facets are larger. In zinc oxide nanostructures, the (0001) planes possess the highest surface energy among the low index planes. As a result, $\mathrm{ZnO}$ grows along the $c$-axis to minimize the surface area of the (0001) planes. The velocity of the crystal growth is related to the different directions as: $[0001]>[01 \overline{1} \overline{1}]>[01 \overline{1} 0]>[01 \overline{1} 1]>[000 \overline{1}]$

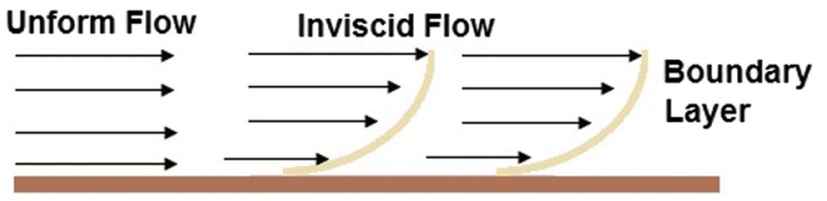

Fig. 6 Illustration of a separation of boundary layer
[23]. The direction with the fastest growth rate leads to the formation of pen holder part with high aspect ratio.

The formation of pen holder part with high aspect ratio is attributed to the [0001] direction with the fastest growth rate [24]. In a general growth habit of crystal, crystal face whose growth rate is fast disappears easily, while faces whose growth rates are slow remain. Thus, $\{01 \overline{1} 1\}$ and $\{01 \overline{1} 0\}$ faces lead consequently to the prism growth on the top of the pen holder.

\section{Conclusion}

A novel pencil-like $\mathrm{ZnO}$ nanowire has been grown on the Si (111) substrate via a modified vapor phase transport method under air atmosphere. A wall (aluminum foil) of $1 \mathrm{~cm}$ in height was inserted between the source and substrate to study the effect of the wall on the morphological and optical properties of the samples. It was found that a separation of the boundary layer on the top of the wall disturbed $\mathrm{Zn}$ diffusion and affected the diameter of the wires. Thus, difference in the diameters of the wires provided pencil-like shape. The obtained $\mathrm{Pl}$ and Raman spectra verify that the optical properties of $\mathrm{ZnO}$ nanostructures grown under different growth conditions are very sensitive to preparation conditions and morphology.

Acknowledgments This work was supported by the Universiti Putra Malaysia through Grant Nos. Fundamental Research Grant Scheme (FRGS) vote: 02-01-14-1469FR.

\section{References}

[1] K. Mahmood, S.B. Park, J. Cryst. Growth 347, 104 (2012)

[2] I.O. Jung, J.Y. Park, S.S. Kim, J. Cryst. Growth 355, 78 (2012)

[3] Q. Ahsanulhaq, A. Umar, Y.B. Hahn, Nanotechnology 18(11), 115603 (2007)

[4] R. Amiruddin, S. Devasia, D.K. Mohammedali, M.S. Kumar, Semicond. Sci. Technol. 30(3), 035009 (2015)

[5] Q.B. Zhang, S.H. Cao, J. Wang, Nano 6, 265 (2011)

[6] A. Kamalianfar, S. Halim, K. Behzad, M.G. Naseri, M. Navasery, F.U. Din, J. Zahedi, K. Lim, S. Chen, H. Sidek, J. Optoelectron, Adv. Mater. 15(3-4), 239 (2013)

[7] F. Jamali-Sheini, R. Yousefi, Ceram. Int. 39(4), 3715 (2013)

[8] Z.W. Pan, Z.R. Dai, Z.L. Wang, Science 291, 1947 (2001)

[9] A.K. Zak, W. Majid, M. Darroudi, R. Yousefi, Mater. Lett. 65(1), 70 (2001)

[10] B.P. Zhang, N.T. Binh, Y. Segawa, Y. Kashiwaba, K. Haga, Appl. Phys. Lett. 84, 586 (2004)

[11] R. Yousefi, M.R. Muhamad, A.K. Zak, Thin Solid Films 518, 5971 (2010)

[12] Z.G. Chen, A. Ni, F. Li, H. Cong, H.M. Cheng, G.Q. Lu, Chem. Phys. Lett. 434(4-6), 301 (2007)

[13] M. Rajalakshmi, A.K. Arora, B.S. Bendre, S. Mahamuni, J. Appl. Phys. 87, 2445 (2000)

[14] N. Hassan, M. Hashim, M. Bououdina, Superlattices Microstruct. 62, 182 (2013) 
[15] R.C. Wang, C.P. Liu, J.L. Huang, S.J. Chen, Appl. Phys. Lett. 86, 251104 (2005)

[16] R. Yousefi, A.K. Zak, Mater. Sci. Semicond. Process. 14(2), 170 (2011)

[17] A. Umar, Y.B. Hahn, Appl. Phys. Lett. 88(17), 173120 (2006)

[18] A. Umar, S.H. Kim, Y.S. Lee, K.S. Nahm, Y.B. Hahn, J. Cryst. Growth 282(1-2), 131 (2005)

[19] J. Gao, X. Zhang, Y. Sun, Q. Zhao, D. Yu, Nanotechnology 21(24), 245703 (2010)

[20] A. Kamalianfar, S.A. Halim, A.K. Zak, Ceram. Int. 40, 3193 (2014)
[21] A. Kamalianfar, S.A. Halim, M.G. Naseri, M. Navasery, F.U. Din, J.A.M. Zahedi, K. Behzad, K.P. Lim, A.L. Moghadam, C.K. Chen, Chin. Phys. B 22, 88103 (2013)

[22] A. Kamalianfar, S.A. Halim, M.G. Naseri, M. Navasery, F.U. Din, J.A.M. Zahedi, K.P. Lim, E.B. Saion, C.K. Chen, A.L. Monfared, Int. J. Electrochem. Sci. 8, 7724 (2013)

[23] K. Vanheusden, C.H. Seager, W.L. Warren, D.R. Tallant, J.A. Voigt, Appl. Phys. Lett. 68, 403 (1996)

[24] G.H. Lee, Mater. Lett. 73, 53 (2012) 